\title{
Expression of $\mathrm{X}$-linked Toll-like receptor 4 signaling genes in female vs. male neonates
}

\author{
David N. O'Driscoll ${ }^{1,2,4}$, Chiara De Santi ${ }^{3}$, Paul J. McKiernann2, Victoria McEneaney ${ }^{4}$, Eleanor J. Molloy ${ }^{1,2,4,5,6}$ and \\ Catherine M. Greene ${ }^{3}$
}

BACKGROUND: Male neonates display poorer disease prognosis and outcomes compared with females. Immune genes which exhibit higher expression in umbilical cord blood (UCB) of females may contribute to the female immune advantage during infection and inflammation. The aim of this study was to quantify expression of Toll-like receptor (TLR) 4 signaling genes encoded on the X-chromosome in UCB from term female vs. male neonates.

METHODS: UCB samples were collected from term neonates ( $n=26$ ) born by elective Caesarean section and whole blood was collected from adults $(n=20)$. Leukocyte RNA was isolated and used in quantitative PCR reactions for IKB kinase $Y(I K K \gamma)$, Bruton's tyrosine kinase (BTK), and IL-1 receptor associated kinase (IRAK) 1. IRAK1 protein was analyzed by Western blot and confocal microscopy.

RESULTS: In neonates there was no significant difference in the relative expression of IKKY or BTK mRNA between genders. IRAK1 gene and protein expression was significantly higher in female vs. male UCB, with increased cytosolic IRAK1 expression also evident in female UCB mononuclear cells. Adults had higher expression of all three genes compared with neonates. CONCLUSION: Increased expression of IRAK1 could be responsible, in part, for sex-specific responses to infection and subsequent immune advantage in female neonates.

0 wing to ontogenic deficiencies in adaptive immunity, neonates depend largely on innate immunity to protect against infection. It is well known that infants display a gender disparity in morbidity and overall mortality, particularly at the borders of viability. For example male neonates have higher rates of infections and sepsis compared with females of matched gestational age, irrespective of higher birth weight distributions (1). Neonatal sepsis is a clinical manifestation of a dysregulated innate immune response to invasive pathogens.

Toll-like receptors (TLRs) are signaling pattern recognition receptors that represent an integral part of innate immunity.
Once activated by recognition of their cognate ligands, TLRs initiate intracellular signaling cascades leading to transcription factor activation e.g., nuclear factor $\kappa \mathrm{B}(\mathrm{NF} \kappa \mathrm{B})$, and induction of cytokine expression. The prototypical TLR, TLR4, is critically important in signaling the inflammatory response to Gram-negative bacteria through recognition of lipopolysaccharide (LPS/endotoxin). TLR4 signals via various intermediate adaptor proteins and kinases (2). One of the most common TLR4 pathways involves signaling via myeloid differentiation factor 88 (MyD88), interleukin-1 receptor-associated kinases (IRAKs) 1 and 4, TNF-receptor-associated factor (TRAF) 6, and inhibitors of NFKB Kinases (IKKs) leading to activation of NFKB; this can be summarized as TLR4/MyD88/IRAKs/ TRAF6/IKKs/NFKB. Bruton's tyrosine kinase (BTK) can participate in this pathway at various levels via individual interactions with TLR4, MyD88, and IRAK1 (3). BTK, and both $I R A K 1$ and IKBKG (the gene that encodes IKK $\gamma$, also known as NEMO) are encoded on the $\mathrm{X}$ chromosome at Xq21 and Xq28, respectively (4).

Sex is determined during embryogenesis by the sex chromosome complement; XX in females and XY in males. The Y chromosome contains $\sim 100$ protein-coding genes, whereas the $\mathrm{X}$ chromosome encodes at least 1,100 including many that are involved in immunity (4). A combination of random silencing of one $\mathrm{X}$ chromosome in females and increased expression levels of dosage-sensitive $\mathrm{X}$ chromosome genes in both sexes achieves dosage compensation. Escape from X chromosome inactivation (XCI) leads to a sex bias in gene expression, and some of these genes are likely to be implicated in the gender differences in certain diseases. Although $\sim 15 \%$ of X-linked genes consistently escape XCI in humans, a further $10 \%$ show variable escape, at least in cell lines $(5,6)$. In females this may confer an advantage in responding to and resolving acute infections.

Various studies have examined expression of X-linked genes involved in TLR signaling in infants and umbilical cord blood (UCB) (7-11). However, to our knowledge, the levels

\footnotetext{
The last two authors are joint senior authors.

${ }^{1}$ Neonatology, National Maternity Hospital, Dublin, Ireland; ${ }^{2}$ Respiratory Research, Department of Medicine, Royal College of Surgeons in Ireland, Education and Research Centre, Beaumont Hospital, Dublin, Ireland; ${ }^{3}$ Clinical Microbiology, Department of Medicine, Royal College of Surgeons in Ireland, Education and Research Centre, Beaumont Hospital, Dublin, Ireland; ' ${ }^{4}$ aediatrics, Academic Centre, Tallaght Hospital, Trinity College, The University of Dublin, Dublin, Ireland; ${ }^{5}$ Neonatology, Coombe Women and Infants' University Hospital, Dublin, Ireland; 'Neonatology, Our Lady's Children's Hospital Crumlin, Dublin, Ireland. Correspondence: David O’Driscoll (odriscd3@tcd.ie) Received 20 July 2016; accepted 3 December 2016; advance online publication 1 March 2017. doi:10.1038/pr.2017.2
} 


\section{Articles $\mid$ o'Driscoll etal.}

of expression of specific X-linked TLR4 signaling intermediate genes in UCB have not been compared between male and female neonates, or adults. In this study, we investigated quantitative differences in BTK, IKK $\gamma$, and IRAK1 mRNA levels in male and female leukocytes from UCB and compared these to levels in adult whole blood. We also explored IRAK1 protein expression in more detail in mononuclear cells isolated from UCB. The data indicate that IRAK1 is expressed more highly in female vs. male UCB and this may underlie, in part, the differences in inflammatory outcomes in infants.

\section{METHODS}

\section{Patient Population and Sample Collection}

Ethical approval for the study was received from the institutional ethics committee at the National Maternity Hospital, Holles Street, Dublin. Informed written consent for blood sampling was obtained in all cases and completed by a single investigator. The study population included the following groups:

1. Adult controls: 10 nonpregnant healthy women and 10 men (laboratory and medical colleagues) aged 20-30 y. Peripheral venous blood $(9 \mathrm{ml})$ was obtained by venepuncture and collected in EDTA-coated vacutainers.

2. Elective lower segment caesarean sections (LSCS): $9 \mathrm{ml} \mathrm{UCB}$ samples ( $n=13$ males, $n=13$ females, $n=26$ ) collected in EDTA-coated vacutainers immediately following elective (no labor) LSCS delivery from uncomplicated term ( $\geq 37 \pm 0 \mathrm{wk}$ ) pregnancies. All elective LSCS were performed under spinal anesthesia. All neonates had an uncomplicated postnatal course (Table 1).

\section{RNA Isolation}

Whole blood samples were centrifuged at $1,800 \mathrm{~g}$ for $20 \mathrm{~min}$ at room temperature to extract plasma and total mononuclear cell fraction (monocytes and lymphocytes). RNA was extracted from $200 \mu \mathrm{l}$ of buffy coat using $750 \mu \mathrm{l} /$ sample Trizol BD reagent (SigmaAldrich, Wicklow, Ireland) as per the manufacturer's protocol. This was followed by phase separation using chloroform $(200 \mu \mathrm{l} /$ sample, Sigma-Aldrich), RNA isolation using isopropanol (500 $\mu \mathrm{l} /$ sample, Sigma-Aldrich) and finally, washing of the RNA pellet in $70 \%$ ethanol, centrifugation to obtain the RNA pellet, and dissolution in $0.1 \%$

Table 1. Characteristics of neonates enrolled in the study

\begin{tabular}{lc}
\hline Demographics & $n=26$ \\
\hline Male & 13 \\
Female & 13 \\
Delivered by elective Caesarean section & 26 \\
Vaginal delivery & 0 \\
Mean gestational age, weeks (SD ${ }^{\mathrm{a}}$ ) & $39 \pm 0(0.63)$ \\
Evidence of labour? & 0 \\
Mean Apgar score at: & \\
$\quad 1$ min & 9.0 \\
5 min & 9.0 \\
10 min & 10.0 \\
Mean age of mother, years (SD) & $35.9(3.49)$ \\
Birthweight (average) & $3.48 \pm 0.27 \mathrm{~kg}$ \\
Postnatal complications? & 0 \\
Admission to NICUb? & 0 \\
\hline
\end{tabular}

aSD, standard deviation; ${ }^{\mathrm{N}} \mathrm{NICU}$, Neonatal Intensive Care Unit. diethyl pyrocarbonate (DEPC)-treated (Sigma-Aldrich) water as per the manufacturer's protocol. Quantification of RNA was performed on an 8-channel Nanodrop-8000 spectrophotometer (Thermo Scientific, Dublin, Ireland). RNA was stored at $-80^{\circ} \mathrm{C}$ until required.

\section{Quantitative PCR}

Equal quantities of RNA were reverse transcribed into cDNA using the Quantitect Reverse Transcription Kit (Qiagen, Manchester, UK) and used for quantitative PCR (qPCR). Primers were designed using BLAST on-line resources. Primer efficiencies were determined from cDNA pooling and serial dilutions $(1,1: 5,1: 25,1: 125$, and 1 : 625) (Table 2). qPCR reactions contained $5 \mu \mathrm{l}$ cDNA, $10 \mu \mathrm{l} \mathrm{SyBr}$ Green mastermix, $3 \mu \mathrm{l}$ PCR $\mathrm{H}_{2} \mathrm{O}$ and $1 \mu \mathrm{l}$ of each a forward and reverse primer (IRAK1, BTK, IKK $\gamma$, or RPLP0) in a $20 \mu$ l volume. Amplification was performed using the LightCycler-480 PCR system (Roche, Dublin, Ireland) at an annealing temperature of $56^{\circ} \mathrm{C}$ for 45 cycles. Quantitative expression of the target genes was determined using the $2^{(-\Delta \Delta C t)}$ method.

\section{Western Blot}

Total protein was isolated from leukocyte samples $(n=5$ male, $n$ $=5$ female). Protein concentrations were determined using the Bicinchoninic acid (BCA) Protein Assay kit (Thermo Scientific) as per the manufacturer's instructions. Samples were denatured by the addition of sample buffer $(62.5 \mathrm{mmol} / \mathrm{l}$ Tris $(\mathrm{pH} 6.8), 2 \%$ sodium dodecyl sulfate (SDS), $0.002 \%$ bromophenol blue, $1.54 \%$ dithiothreitol, $1 \%$ sucrose, $1 \mathrm{mmol} / \mathrm{l}$ EDTA in $\mathrm{dH} 20)$, and were boiled at $95^{\circ} \mathrm{C}$ for $5 \mathrm{~min}$ prior to separation by $12.5 \%$ sodium dodecyl sulfate-polyacrylamide gel electrophoresis (SDS-PAGE). Samples were transferred to a polyvinylidene-fluoride (PVDF) membrane (Thermo Scientific) and incubated with mouse monoclonal IRAK1 antibody (Santa Cruz sc-55530, 1:2000, Heidelberg, Germany) in 1\% bovine serum albumin (BSA)-tris-buffered saline (TBS) and 0.1\% Tween-20 overnight at $4^{\circ} \mathrm{C}$. Following a series of washes, membranes were incubated with rabbit polyclonal glyceraldehyde 3-phosphate dehydrogenase (GAPDH) (Santa Cruz Fl-335, 1:2000, Heidelberg, Germany) and washed as before. Membranes were incubated with horseradish peroxidase-conjugated IgG secondary antibodies (antimouse Cell Signaling 7076, or antirabbit Cell Signaling 7074, both 1:2000, Dublin, Ireland). Following washing, signal detection was determined using HRP substrate on the Syngene G:Box chemi-XL gel documentation system. Densitometry was analyzed using GeneTools software.

\section{Peripheral Blood Mononuclear Cell Isolation}

Freshly collected UCB samples ( $n=3$ male, $n=3$ female) were diluted in $\mathrm{dPBS}$ at room temperature. UCB samples were overlayered onto Lymphoprep (Stemcell Technologies, Cambridge, UK) and centrifuged at $800 \mathrm{~g}$ for $10 \mathrm{~min}$ at room temperature (RT). The mononuclear cell interphase band containing monocytes and lymphocytes was aspirated and washed in Hank's Balanced Salt Solution (HBSS) medium and resuspended in Roswell Park Memorial Institute (RPMI) 1640. 2X Freezing Media (10\% dimethylsulfoxide (DMSO), 40\% fetal calf serum (FCS), 50\% RPMI 1640) was added to cell suspensions. Samples were transferred into cryovials and placed at $-80^{\circ} \mathrm{C}$. After $24 \mathrm{~h}$, cryovials were transferred into liquid nitrogen where they were stored until further use.

\section{Confocal Microscopy}

Isolated peripheral blood mononuclear cells (PBMCs) were incubated for $30 \mathrm{~min}$ in $4 \%$ paraformaldehyde (Sigma-Aldrich) in 1XPBS, washed in 1XPBS, and permeabilised with $0.5 \%$ Triton X-100 in $1 \mathrm{XPBS}$ for $10 \mathrm{~min}$. Cells were washed twice in $1 \mathrm{XPBS}$ and stained with $50 \mu \mathrm{g} / \mathrm{ml}$ fluorescent rhodamine phalloidin conjugate solution (Thermo Scientific) to stain F-actin in 1XPBS for $40 \mathrm{~min}$ at room temperature and washed three times with 1XPBS to remove unbound phalloidin conjugate. Nunc 8 well chamber slides (Thermo Scientific) were coated in poly-L-Lysine (Sigma-Aldrich) at $37^{\circ} \mathrm{C}$ for $30 \mathrm{~min}$ and left to dry at room temperature. Isolated PBMCs were resuspended and allowed to adhere to the slides overnight. Chamber slides were incubated with blocking buffer (4\% BSA in 1XPBS) for $30 \mathrm{~min}$ at $37^{\circ} \mathrm{C}$ to minimize nonspecific absorption of the antibodies. The slide was incubated overnight at $37^{\circ} \mathrm{C}$ in primary IRAK1 antibody (Santa Cruz, sc-55530, 1:250, Heidelberg, Germany) in 1XPBS, the antibody was removed and the slide was washed twice 
Table 2. Primer sequences and efficiency values

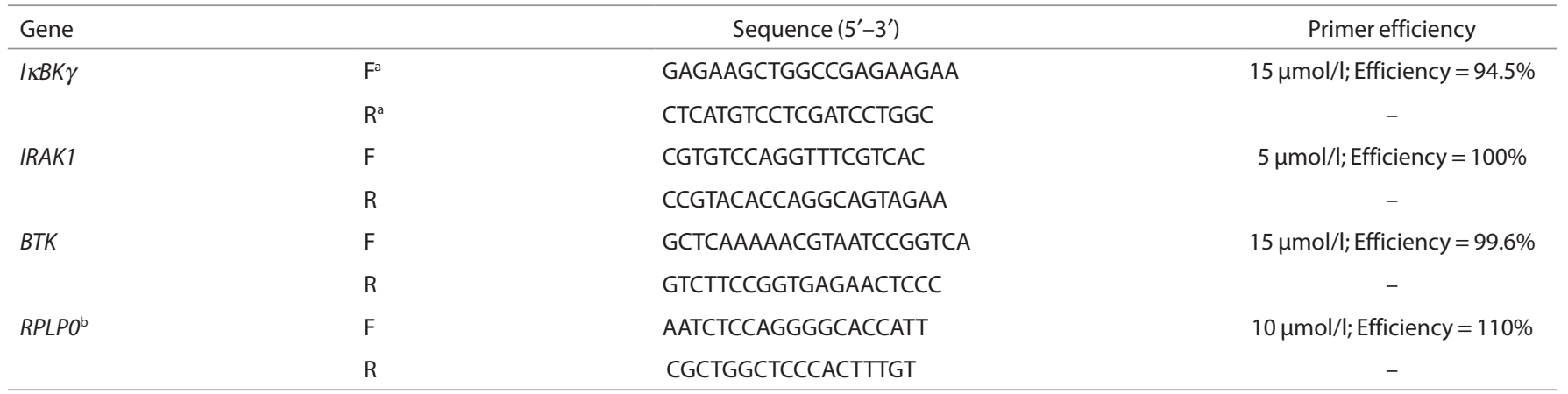

${ }^{\mathrm{a}}$, forward; R, Reverse. ${ }^{\mathrm{b}} \mathrm{RPLPO}$, ribosomal protein lateral stalk subunit PO.

BTK, Bruton's tyrosine kinase; IKK $\gamma$, IKB kinase $\gamma$; IRAK1, interleukin-1 receptor associated kinase 1; qPCR, quantitative PCR; UCB, umbilical cord blood.

in 1XPBS. Cells were incubated for $2 \mathrm{~h}$ at room temperature in goat antimouse DyLight-488 secondary antibody (abcam, ab96879, 1:500, Cambridge, UK) in 1XPBS, washed, fluorescent mounting media containing 4,6-diamidino-2-2-phenylindole (DAPI) was added and a coverslip was placed onto the slide and sealed for imaging. Images were captured using a Zeiss 510 meta confocal microscope at X40 magnification and X 4 zoom. Scans were performed at $1 \mu \mathrm{m}$ interval depths through the fixed cells, and single or merged images are presented as XY single planes through the mid-section of the images unless otherwise specified.

\section{Statistical Analyses}

All statistical analyses were performed using GraphPad Prism 4.0 software package (San Diego, CA). Significance was assumed for values of $P<0.05$ (Student's $t$-test, ANOVA).

\section{RESULTS}

\section{Patient Demographics}

The mean duration of pregnancy for this cohort $(n=26)$ was $39 \pm 0 \mathrm{wk}(0.63 \mathrm{SD})$. The indications for elective LSCS were breech/transverse lie, previous LSCS, and previous obstetric perineal (third degree) tear and this maternal cohort did not labor (no rupture of membranes/premature rupture of membranes, and no cervical dilatation). Recruited mothers were of Caucasian ethnicity and did not have a history of acute or chronic disease. Delivery of all recruited neonates by LSCS was uncomplicated. All neonates had Apgar scores of 9 at 1 and $5 \mathrm{~min}$, and 10 at $10 \mathrm{~min}$. Average birth weight was $3.48 \pm 0.27$ (SD) $\mathrm{kg}(3.56 \mathrm{~kg} \pm 0.33(\mathrm{SD})$ in male neonates and $3.37 \mathrm{~kg} \pm$ 0.29 (SD) in female neonates; $P=0.127$ ). All neonates had an uncomplicated postnatal course and were discharged with their mothers.

\section{Neonates have Lower BTK, IKK $\gamma$, and IRAK1 Gene Expression Compared with Adults}

The X chromosome encodes a large number of genes involved in immunity (4). Here the levels of expression of three X-linked genes involved in TLR4 signaling were quantified in leukocytes from neonatal UCB and adult whole blood. Figure 1 shows the relative expression levels of BTK, IKK $\gamma$, and IRAK1 mRNA in cord blood vs. adult whole blood quantified by qPCR. For all three genes neonates had significantly lower levels compared with adults (Figure 1, $P<0.0001$ ).

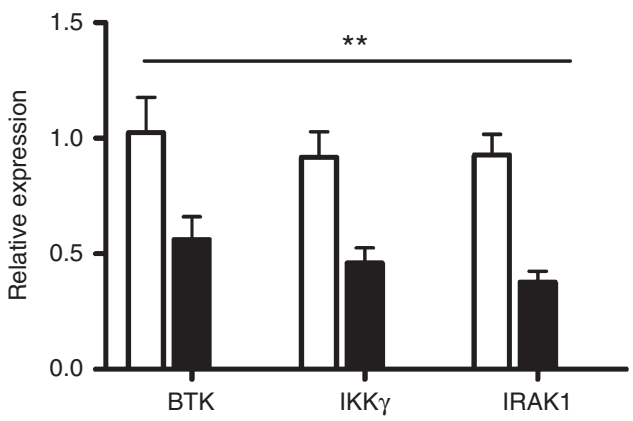

Figure 1. Relative expression of BTK, IKK $\gamma$, and IRAK1 in adult blood vs. UCB. RNA was isolated from the buffy coat of UCB or adult whole blood and used in BTK, IKK $\gamma$, and IRAK1 qPCRs in triplicate ( $n=20$ per group). Relative gene expression (mean \pm S.E.) was calculated for these target genes by comparison with the reference gene RPLPO using the $2^{-\triangle \Delta C t}$ method. ${ }^{* *} P<0.0001$ ANOVA with Bonferroni's multiple comparison tests. White $=$ adults, black $=$ neonates. BTK, Bruton's tyrosine kinase; IKK $\gamma$, IKB kinase $\gamma$; IRAK1, interleukin-1 receptor associated kinase 1 ; qPCR, quantitative PCR; UCB, umbilical cord blood.

\section{Sex-specific BTK, IKK $\gamma$, and IRAK1 Gene Expression Levels in Adults and Neonates}

Next, we stratified the samples by sex in order to determine if there are any sex-specific differences in expression of BTK, IKK $\gamma$, or IRAK1 mRNA in leukocytes from adult blood or UCB. Figure 2a shows that although levels of each of the three transcripts tended to be lower in adult females vs. males, there was no significant difference overall. In UCB samples there was no difference in BTK expression between the sexes (Figure $2 \mathbf{b}$ ). Levels of IKK $\gamma$ mRNA were slightly higher in female neonates (ns, $P=0.119$ ) however IRAK1 relative gene expression was significantly higher in female neonates compared with male neonates $(P=0.0198)$.

\section{IRAK1 Protein Levels in Female vs. Male Neonates}

In order to investigate whether IRAK1 protein, like IRAK1 mRNA expression, was also increased in female UCB we performed western immunoblotting for IRAK1 on cells isolated from male and female UCB that were previously analyzed by qPCR. IRAK1 protein levels were variable but detectable 


\section{Articles | o'Driscolletal.}

in whole cell extracts isolated from leukocytes isolated from female UCB (Figure 3). IRAK1 protein was barely, if at all, detectable in the male samples.

\section{IRAK1 Levels and Localization in Female vs. Male PBMCs}

IRAK1 is a cytosolic protein that participates in TLR4 and other TLR signaling pathways. To further confirm our observations regarding increased IRAK1 mRNA and protein expression in female vs. male UCB leukocytes, we next isolated mononuclear cells from additional cord blood samples and used confocal microscopy, which is more sensitive than western blotting, to visualize IRAK1 localization within these cells. Figure 4 shows that compared with male mononuclear cells, IRAK1 protein is more highly expressed in female cord blood mononuclear cells where it colocalizes with F-actin in the cytosol.

\section{DISCUSSION}

Females have a well-recognized survival advantage compared with males throughout the entire lifecycle. The female immune advantage is particularly evident during the newborn period, as evidenced by Ulizzi et al. who described a generalized male overmortality in the early period of extrauterine life (12).
Here we assessed the expression levels of three X-linked TLR4 signaling genes in female vs. male UCB in order to observe whether differences exist in transcript levels between the sexes. Having detected higher IRAK1 gene and protein expression in female leukocytes, we further demonstrated increased cytosolic IRAK1 protein expression in female vs. male mononuclear cells isolated from UCB. The data presented here support our hypothesis that expression of TLR4 signaling intermediates encoded on the $\mathrm{X}$ chromosome might be altered in monocytes from female UCB. Based on these observations we speculate that increased basal expression of IRAK1 could contribute to sex-specific responses to infection and an immune advantage in female neonates.

Both monocytes and neutrophils are major cells of the innate immune response; in this study we were interested in the behavior of monocytes. These cells originate from precursors in the bone marrow, and circulate in the bloodstream, until they are attracted to infection or inflammatory signals where they differentiate into macrophage or dendritic cell populations. The monocyte/macrophage lineage of myeloid cells has three primary roles to this effect: phagocytosis, antigen presentation, and immunomodulation. Our initial observations a

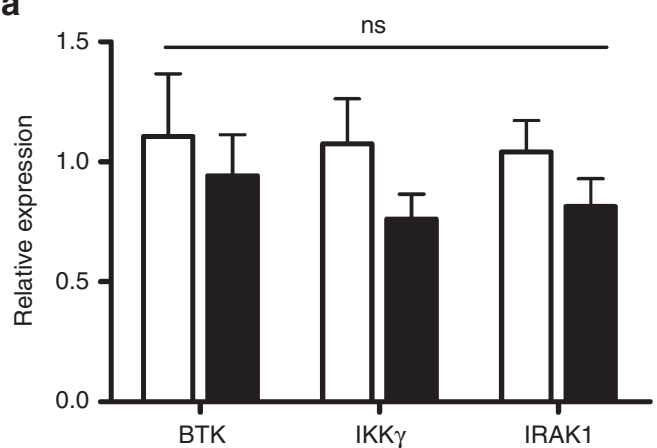

b

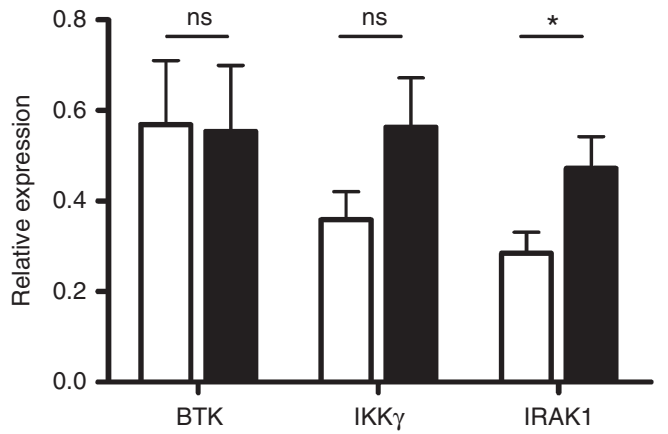

Figure 2. Sex-specific BTK, IKK $\gamma$, and IRAK1 gene expression levels in adults and neonates. RNA was isolated from the buffy coat of male and female (a) adult whole blood or (b) UCB ( $n=10$ per group) and used in triplicate BTK, IKK $\gamma$, and IRAK1 qPCRs. Relative gene expression (mean \pm S.E.) was calculated for these target genes by comparison with the reference gene $R P L P O$ using the $2^{-\triangle \triangle C t}$ method. ${ }^{*} P=0.0198$ unpaired $t$-test, ANOVA. White $=$ males, black $=$ females. BTK, Bruton's tyrosine kinase; IKK $\gamma$, IKB kinase $\gamma$; IRAK1, interleukin-1 receptor associated kinase 1; qPCR, quantitative PCR; UCB, umbilical cord blood.

a

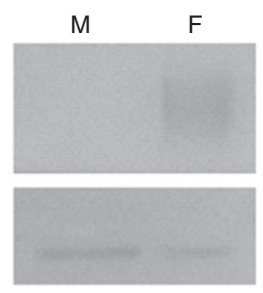

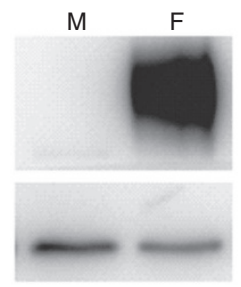

IRAK1 (80 kDa)

GAPDH $(37 \mathrm{kDa})$

b

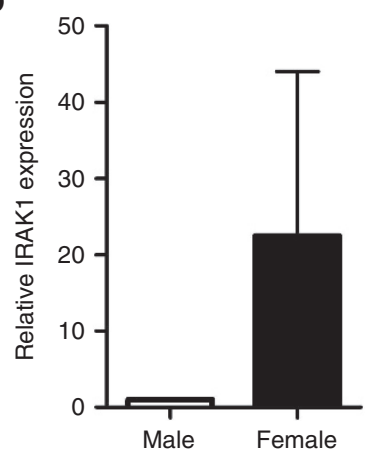

Figure 3. IRAK1 protein levels in female vs. male neonates. Protein extracts $(20 \mu \mathrm{g})$ from the buffy coat of male and female UCB ( $n=5$ per group) were electrophoresed on 10\% SDS-PAGE, transferred onto nitrocellulose and probed with anti-IRAK1 and anti-GAPDH antibodies by western blotting. (a) Representative blots $(n=2)$ and (b) densitometric analysis of IRAK1 protein expression $(n=5$ per group, mean \pm S.E.) normalized to GAPDH for all samples. $\mathrm{M}=$ males, $\mathrm{F}$ = females. GAPDH, glyceraldehyde 3-phosphate dehydrogenase; IRAK1, interleukin-1 receptor associated kinase 1; SDS, sodium dodecyl sulfate; PAGE, polyacrylamide gel electrophoresis; qPCR, quantitative PCR; UCB, umbilical cord blood. 


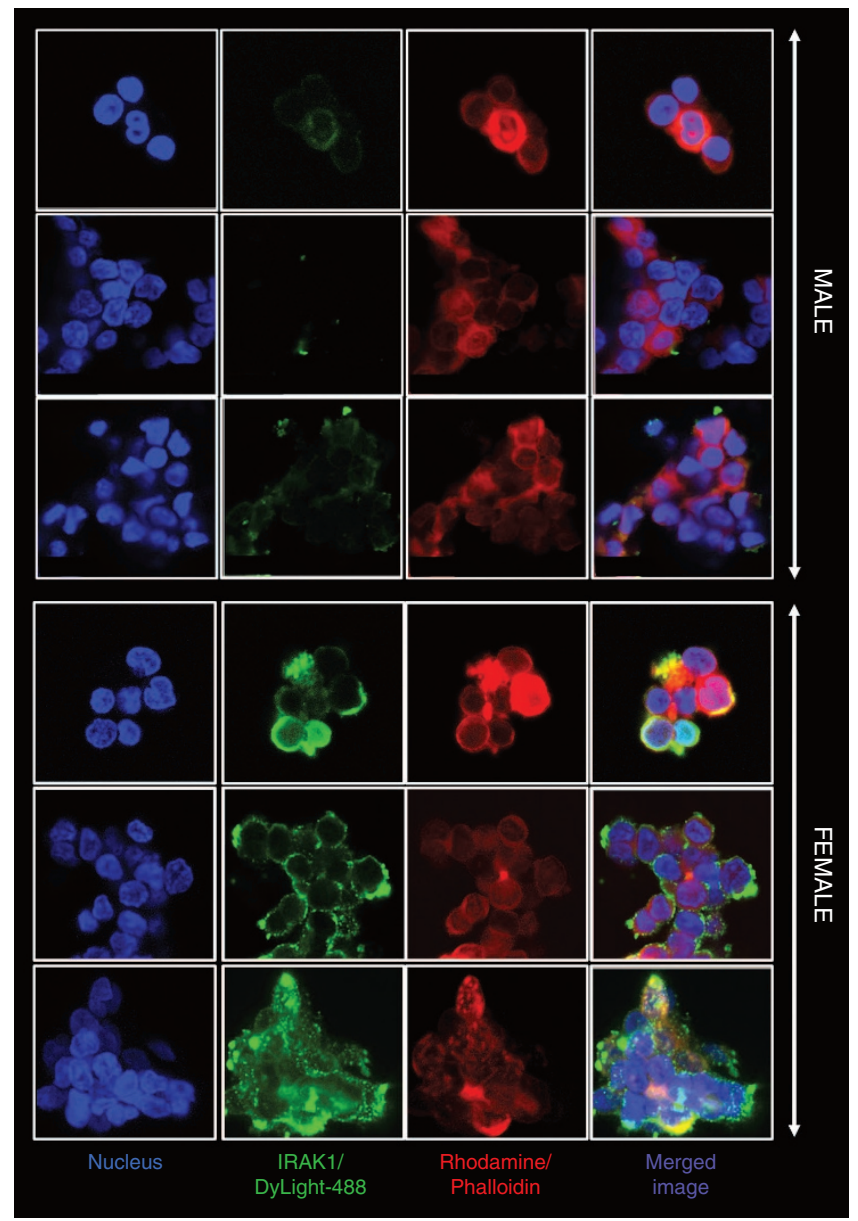

Figure 4. Confocal microscopy images of IRAK1 in PBMCs. PBMCs were isolated from male and female UCB ( $n=3$ per group), fixed, permeabilized, and labelled with DAPI (blue, nucleus), rhodamine phalloidin conjugate solution (red, cytosol) and anti-IRAK1 antibody primary and secondary antibodies (green). Images were captured using a Zeiss 510 meta confocal microscope at X40 magnification and X4 zoom. Scans were performed at $1 \mu \mathrm{m}$ interval depths through the fixed cells and single or merged images are presented as XY frontal focal planes. DAPI, 4,6-diamidino-2-2-phenylindole; IRAK1, interleukin-1 receptor associated kinase 1; PBMCs, peripheral blood mononuclear cells; UCB, umbilical cord blood.

pointed to a difference in IRAK1 expression in female vs. male UCB leukocytes. In order to focus more closely on monocytes, we performed subsequent analyses on total mononuclear rather than granulocytic cells. Within the mononuclear fraction lymphocytes as well as monocytes are also present. Thus our observations also potentially relate to adaptive immune responses, however these are not evident in very early life.

The $\mathrm{X}$ chromosome encodes many genes that are involved in immune functions such as as TLR7, CD40 ligand, the IL-1 receptor accessory protein, IL-2 receptor $\gamma$, and the IL-9 receptor, amongst many others (4). In females protein-coding genes on the X chromosome are susceptible to XCI however despite chromosome-wide transcriptional silencing of one $\mathrm{X}$ chromosome, some genes remain expressed from both the active and inactive $\mathrm{X}$ alleles, resulting in a double gene dosage. It has been estimated that in women, $\sim 15 \%$ of $\mathrm{X}$-linked genes escape XCI and are biallelically expressed (13). Interestingly this selection of genes includes genes encoding for several components of the TLR4 pathway that are essential for NFKB signaling. Amongst these is a subset of genes with specific roles in innate immunity, such as BTK located at Xq22.1, and a cluster of genes that maps to Xq28, where IKBKG and IRAK1 are located (5). Thus, higher expression of these genes in females compared with males may confer an advantage in responding to and resolving acute infections and could be implicated in gender differences in certain diseases.

Neonates depend largely on the innate immune system as the first line of defense against microbial invasion. The TLR4 pathway recognizes and responds to LPS, and abnormal LPS responses are associated with many diseases in infants such as sepsis, bronchopulmonary dysplasia, necrotizing enterocolitis and cerebral white matter damage with resultant cerebral palsy-all of which are noted to preferentially affect male neonates (14-18). Immunologically immature neonates suffer the highest incidence of sepsis (19-22). Maturational differences in the immune response can affect a neonate's ability to mount an appropriate inflammatory response to infection and clear pathogens $(19,21)$. Clinical studies suggest that a hypo-inflammatory state is associated with an increased risk of secondary infections and mortality in neonates $(19,23)$. Evaluation of downstream signaling components in the TLR4/MyD88 pathway has revealed differential expression of signal transducing proteins based on immune maturation status (19). Yan et al. demonstrated that MyD88 expression is significantly reduced in neonatal monocytes and is associated with decreased tumor necrosis factor- $\alpha$ production following low-dose LPS stimulation (24). IRAK1 is also a key signal transducer in the TLR4 pathway and increased expression of IRAK1, as observed here in female UCB, may enhance TLR4 functional responses, thereby facilitating a more effective innate immune response in the face of infection. It would be interesting to determine the effect of LPS on IRAK1, IKBKG, and BTK gene expression; future studies will attempt to investigate this. What also remains to be determined is the mechanism(s) by which IRAK1 expression is increased. Future studies should investigate the potential roles of epigenetic influences (e.g., promoter methylation) and differential transcription factor activation. It is also possible that neonatal TLR4 pathway ontogeny may be responsible for lower IRAK1 expression in male neonates. IRAK1 is a validated target of miR-146a (25) and decreased miR-146a levels may be accountable for the observed effect of IRAK1 overexpression in females, however this remains speculative. Importantly neonates are subjected to very high levels of sex steroids resulting from pregnancy, in particular $17 \beta$-estradiol, which might influence their immune system. Therefore $17 \beta$-estradiol and progesterone may also have a role here, however addressing these questions are beyond the scope of this study.

Here we saw no significant difference in BTK and IKK $\gamma$ transcript levels in female vs. male UCB, although IKK $\gamma$ mRNA levels showed a trend toward being higher in females $(P=0.119)$, suggesting that this may be true in a larger powered study. Indeed both IRAK1 and IKBKG are closely located on Xq28, 


\section{Articles | o'Driscoll tetal.}

a region known to display XCI escape (5). BTK is involved in TLR4 signaling and has been shown to interact with TLR4, MyD88, and IRAK1 to facilitate signal transduction in certain instances (3). The absence of any observable difference in BTK mRNA levels in this pilot study indicates that its expression in UCB may not be gender-related. We also saw no significant difference in BTK mRNA levels in male vs. female adult whole blood. Surprisingly, none of the X-linked genes studied here were expressed at a higher level in adult females compared with males, however the sample size was small.

It is important to consider that IRAK1 variant haplotypes exist with different functional activities in vivo. Generally the IRAK1 haplotype with increased activity (single nucleotide polymorphism in which $\mathrm{T}$ is replaced with $\mathrm{C}$ at nucleotide 1,595 within exon 12 of the IRAK1 gene) is associated with poorer outcomes in sepsis in humans $(26,27)$. Yet females heterozygous for the "high-activity" or wild-type IRAK1 haplotypes show no adverse clinical consequences. In our study cohorts, it is not known which IRAK1 variants are present. Preclinical data describes both protective and detrimental effects of IRAK1 deficiency in models of sepsis $(28,29)$. In an adult mouse model of caecal ligation and puncture-induced sepsis, IRAK1 deficiency can protect against sepsis (28). However those authors also reported that IRAK1 levels have been shown to have both protective and deleterious effects in other animal injury models. Overall, it appears that the effect is model-dependent.

In this study adults expressed higher levels of all three genes compared with neonates. Various studies have reported overall reduced immune-related gene expression in neonates compared with adults. For example, expression of Transforming Growth Factor- $\beta 1$, macrophage inflammatory protein- $1 \alpha$, and IL-8 protein were all shown to be lower following agoniststimulation in human neonatal vs. adult mononuclear cells; however that effect was thought to be post-transcriptional (30). De Wit et al. reported that CpG-induced Interferon- $\gamma$ mRNA and protein in plasmacytoid dendritic cells was dramatically impaired in cord vs. adult blood, and described how these cells are intrinsically deficient in CpG-induced IFN- $\gamma$ production at birth (31). The gene encoding TLR3 has also been shown to display developmental regulation in dendritic cells derived from cord blood (32). Novel data presented here indicate that $B T K, I K B K G$, and IRAK1 are also expressed at lower levels in neonates compared with adults.

One limitation of this observational study is the small number of patients in all groups. Future work should aim to cover a broader clinical cohort, including preterm infants. We are also aware that in this study the entire patient cohort consisted of neonates born by Caesarean section without labor. Since labor promotes neonatal neutrophil survival and LPSresponsiveness, for example (33), it would be interesting to determine whether it also affects TLR4 functional responses in PBMCs. In conclusion the novel observations from this study regarding increased IRAK1 gene and protein expression in female vs. male cord blood mononuclear cells provide a sound basis for future mechanistic and disease-related studies in larger cohorts of term and preterm infants.

\section{ACKNOWLEDGMENTS}

The authors thank all of the parents and controls who consented to donate blood samples to this project. The authors also thank the Operating Theatre staff at the National Maternity Hospital, Holles Street for their clinical assistance.

\section{STATEMENT OF FINANCIAL SUPPORT}

This study was supported by Health Research Board, European Respiratory Society/GlaxoSmithKline.

Disclosure: The authors declare no conflicts of interest. The authors do not have any financial ties to products in the study.

\section{REFERENCES}

1. Peacock JL, Marston L, Marlow N, Calvert SA, Greenough A. Neonatal and infant outcome in boys and girls born very prematurely. Pediatr Res 2012;71:305-10.

2. Greene C. The Toll-like Receptor Family. In: Toll-Like Receptors in Diseases of the Lung: Bentham eBooks. 2012. p. 3-11.

3. O'Neill LA. When signaling pathways collide: positive and negative regulation of toll-like receptor signal transduction. Immunity 2008;29:12-20.

4. Libert C, Dejager L, Pinheiro I. The X chromosome in immune functions: when a chromosome makes the difference. Nat Rev Immunol 2010;10:594604.

5. Carrel L, Willard HF. X-inactivation profile reveals extensive variability in X-linked gene expression in females. Nature 2005;434:400-4.

6. Deng X, Berletch JB, Nguyen DK, Disteche CM. X chromosome regulation: diverse patterns in development, tissues and disease. Nat Rev Genet 2014;15:367-78.

7. Kallionpää H, Laajala E, Öling V, et al.; DIABIMMUNE Study Group. Standard of hygiene and immune adaptation in newborn infants. Clin Immunol 2014;155:136-47.

8. Plebani A, Soresina A, Rondelli R, et al.; Italian Pediatric Group for XLAAIEOP. Clinical, immunological, and molecular analysis in a large cohort of patients with X-linked agammaglobulinemia: an Italian multicenter study. Clin Immunol 2002;104:221-30.

9. Charrier E, Cordeiro P, Cordeau M, et al. Post-transcriptional down-regulation of Toll-like receptor signaling pathway in umbilical cord blood plasmacytoid dendritic cells. Cell Immunol 2012;276:114-21.

10. Ørstavik KH, Kristiansen M, Knudsen GP, et al. Novel splicing mutation in the NEMO (IKK-gamma) gene with severe immunodeficiency and heterogeneity of X-chromosome inactivation. Am J Med Genet A 2006;140:31-9.

11. Sakowicz A, Hejduk P, Pietrucha T, et al. Finding NEMO in pre-eclampsia. Am J Obstet Gynecol 2016;214:538.e1-7.

12. Ulizzi L, Zonta LA. Sex differential patterns in perinatal deaths in Italy. Hum Biol 2002;74:879-88.

13. Berletch JB, Yang F, Xu J, Carrel L, Disteche CM. Genes that escape from X inactivation. Hum Genet 2011;130:237-45.

14. O'Hare FM, William Watson R, Molloy EJ. Toll-like receptors in neonatal sepsis. Acta Paediatr 2013;102:572-8.

15. Elsmén E, Hansen Pupp I, Hellström-Westas L. Preterm male infants need more initial respiratory and circulatory support than female infants. Acta Paediatr 2004;93:529-33.

16. Carter BM, Holditch-Davis D. Risk factors for necrotizing enterocolitis in preterm infants: how race, gender, and health status contribute. Adv Neonatal Care 2008;8:285-90.

17. Fleiss B, Gressens P. Tertiary mechanisms of brain damage: a new hope for treatment of cerebral palsy? Lancet Neurol 2012;11:556-66.

18. Kraemer S. The fragile male. BMJ 2000;321:1609-12.

19. Maddux AB, Douglas IS. Is the developmentally immature immune response in pediatric sepsis a recapitulation of immune tolerance? Immunology 2015;145:1-10.

20. Randolph AG, McCulloh RJ. Pediatric sepsis: important considerations for diagnosing and managing severe infections in infants, children, and adolescents. Virulence 2014;5:179-89.

21. Sadeghi K, Berger A, Langgartner M, et al. Immaturity of infection control in preterm and term newborns is associated with impaired toll-like receptor signaling. J Infect Dis 2007;195:296-302. 
22. Ballow M, Cates KL, Rowe JC, Goetz C, Desbonnet C. Development of the immune system in very low birth weight (less than $1500 \mathrm{~g}$ ) premature infants: concentrations of plasma immunoglobulins and patterns of infections. Pediatr Res 1986;20:899-904.

23. López-Collazo E, del Fresno C. Pathophysiology of endotoxin tolerance: mechanisms and clinical consequences. Crit Care 2013;17:242.

24. Yan SR, Qing G, Byers DM, Stadnyk AW, Al-Hertani W, Bortolussi R. Role of MyD88 in diminished tumor necrosis factor alpha production by newborn mononuclear cells in response to lipopolysaccharide. Infect Immun 2004;72:1223-9.

25. Taganov KD, Boldin MP, Chang KJ, Baltimore D. NF-kappaB-dependent induction of microRNA miR-146, an inhibitor targeted to signaling proteins of innate immune responses. Proc Natl Acad Sci USA 2006;103:12481-6.

26. Arcaroli J, Silva E, Maloney JP, et al. Variant IRAK-1 haplotype is associated with increased nuclear factor-kappaB activation and worse outcomes in sepsis. Am J Respir Crit Care Med 2006;173:1335-41.

27. Toubiana J, Courtine E, Pène F, et al. IRAK1 functional genetic variant affects severity of septic shock. Crit Care Med 2010;38:2287-94.
28. Chandra R, Federici S, Bishwas T, et al. IRAK1-dependent signaling mediates mortality in polymicrobial sepsis. Inflammation 2013;36: 1503-12.

29. Chandra R, Federici S, Németh ZH, et al. Cellular mosaicism for X-linked polymorphisms and IRAK1 expression presents a distinct phenotype and improves survival following sepsis. J Leukoc Biol 2014;95:497-507.

30. Chang M, Suen Y, Lee SM, et al. Transforming growth factor-beta 1, macrophage inflammatory protein-1 alpha, and interleukin-8 gene expression is lower in stimulated human neonatal compared with adult mononuclear cells. Blood 1994;84:118-24.

31. De Wit D, Olislagers V, Goriely S, et al. Blood plasmacytoid dendritic cell responses to $\mathrm{CpG}$ oligodeoxynucleotides are impaired in human newborns. Blood 2004;103:1030-2.

32. Porrás A, Kozar S, Russanova V, et al. Developmental and epigenetic regulation of the human TLR3 gene. Mol Immunol 2008;46:27-36.

33. Molloy EJ, O'Neill AJ, Grantham JJ, et al. Labor promotes neonatal neutrophil survival and lipopolysaccharide responsiveness. Pediatr Res 2004;56:99-103. 